\title{
Perspective
}

PERSPECTIVE Actualité en histoire de l'art

1 | 2008

Antiquité/Moyen Âge

\section{Théâtre et céramique d'Italie méridionale et de Sicile : de nouvelles perspectives}

Alexa Piqueux

\section{OpenEdition}

1 Journals

Édition électronique

URL : http://journals.openedition.org/perspective/3496

DOI : 10.4000/perspective.3496

ISSN : 2269-7721

Éditeur

Institut national d'histoire de l'art

Édition imprimée

Date de publication : 31 mars 2008

Pagination : 67-72

ISSN : 1777-7852

Référence électronique

Alexa Piqueux, "Théâtre et céramique d'Italie méridionale et de Sicile : de nouvelles perspectives »,

Perspective [En ligne], 1 | 2008, mis en ligne le 31 mars 2018, consulté le 01 octobre 2020. URL : http:// journals.openedition.org/perspective/3496; DOI : https://doi.org/10.4000/perspective.3496 


\section{t héâtre et céramique d'italie méridionale et de Sicile : de nouvelles perspectives}

\section{Alexa Piqueux}

- Oliver Taplin , Pots and Plays. Interactions between Tragedy and Greek Vase-Painting of the Fourth Century B.C., 1 os a ngeles, the J. Paul Getty Museum, 2007. 320 p., 85 fig. en n. et b et 94 fig. en coul. iSBN : 978-0-89236-807-5; $\$ 75$.

- a lan Hug Hes, " c omedy in Paestan Vase Painting ", dan Oxford Journal of Archaeology, 22/3, Blackwell (Oxford), 2003 p. 281-301. iSSN : 0262-5253; \$42, 63.

- a ngela pon Trandolfo , "Dioniso e personaggi fliacici nelle immagini pestane ", dans Ostraka: Rivista di antichità, 9/1, l offredo (Naples), 2000, p. 117-134. iSSN : 1122 $259 \mathrm{X} ; 34 €$.

- E. G.D. $r$ obinson , « Reception of c omic theatre amongst the indigenous South italians ", dans c raig bar ker, 1 esley b eaumon T, Elizabeth bollen éd., Festschrift in Honour of J. Richard Green, (Mediterranean Archaeology, 17) Sydney, u niversity of Sydney, 2004 (2006), p. 193-212. 327 p., 45 fig. iSSN : 1030-8482; \$75.

l es rapports entre littérature et céramique ont fait ces dernières décennies l'objet d'intenses réflexions, qui ont abouti à la reconnaissance de l'autonomie de ces deux arts, mais malheureusement bien souvent aussi à leur traitement séparé de la part de savants spécialisés en philologie ou en histoire de l'art. l e théâtre grec, art visuel tout autant que rhétorique, a souffert plus qu'aucun autre genre de ce cloisonnement. Dans ce contexte, le très beau livre d'Oliver taplin, Pots and Plays, qui s'adresse autant aux spécialistes de l'a ntiquité qu'à un public plus large, est fort bienvenu. c ent neuf vases du iv ${ }^{\mathrm{e}}$ siècle av. J.-c . provenant en majorité d'a pulie, mais aussi d'a ttique, de l ucanie, de Sicile et de campanie y sont étudiés ${ }^{1}$. Oliver taplin, philologue de formation, a ouvert la voie à la fin des années 1970 à une étude des textes dramatiques antiques prenant en compte la réalité concrète de leur mise en scène et a réexaminé avec succès dans les années 1990 les liens existant entre la comédie athénienne et les vases italiotes à sujet comique dits "phlyaques $"^{2}$. il propose ici une nouvelle approche de l'empreinte laissée par le théâtre tragique - essentiellement celui des trois grands dramaturges athéniens du $v^{e}$ s. av. J.-c ., Eschyle, Sophocle et Euripide - sur les scènes à caractère mythologique de la céramique italiote du iv ${ }^{\mathrm{e}} \mathrm{s}$. av. J.-c . qui, à l'exception de quelques peintures siciliennes incluant notamment un appareil scénique $\left(\mathrm{n}^{\circ} 22,103-106\right)$, ne présentent à première vue aucun indice évident de théâtralité.

le livre comprend deux parties. la première, intitulée "Setting the Scenes ", constitue une introduction de 46 pages où sont présentés les corpus dramatiques et céramiques ainsi que les enjeux de l'ouvrage et la méthode adoptée par l'auteur. l a seconde partie, "t he Pots", est organisée en cinq chapitres. l es deux premiers correspondent aux ouvres d'Eschyle et de Sophocle ; les deux suivants, aux tragédies d'Euripide, entièrement conservées ou fragmentaires, qui semblent avoir été particulièrement appréciées en italie. l e dernier chapitre regroupe les scènes peintes mises en relation avec une œuvre tragique indéterminée. c haque vase fait l'objet d'une analyse d'une ou deux pages, accompagnée d'une reproduction de grand format, le plus souvent d'excellente qualité. la connaissance intime que l'auteur a des textes tragiques et la finesse de ses analyses iconographiques font de la lecture de cet ouvrage très stimulant un véritable plaisir pour les philologues comme pour les historiens de l'art.

l es enjeux de cet examen des liens existant entre théâtre tragique et peinture de vases relèvent à la fois de l'histoire de l'art et de l'histoire culturelle de la Grande Grèce. l es images antiques ont longtemps été considérées comme un reflet de la littérature contemporaine, qui les aurait inspirées. c'est un peu dans cet esprit qu'avait été conçu au début des années 1970 l'ouvrage d'a rthur Dale trendall et t homas Bertram l onsdale Webster, Illustrations of Greek Drama (l ondres, 1971), qui faisait jusqu'à présent référence. Refusant à juste titre cette supériorité $\mathrm{du}$ texte sur l'image, les travaux des trente dernières années ont abouti à considérer trop souvent ces deux médias comme entièrement indépendants. l'aporie à laquelle conduisent les perspectives des "philodramatistes " comme des "iconocentristes " pour l'analyse des scènes mythologiques a été soulignée à la fin des années 1990 par l uca Giuliani, qui forge ces deux néologismes ${ }^{3}$. Proposant dans son sillage une voie médiane entre ces deux positions extrêmes 
tout en se démarquant de ses conclusions, Oliver taplin prend le parti de s'attacher moins à la culture du peintre qu'aux processus de remémoration et d'association d'idées touchant celui qui regarde l'image peinte d'un mythe qu'il a déjà vu représenté au théâtre. l'auteur, qui considère que les représentations tragiques constituentl'une desformes majeures de narration du mythe dans la Grèce du iv ${ }^{\text {e }}$ siècle av. J.-c ., avance l'idée que la connaissance d'une tragédie particulière peut enrichir la lecture d'une image, dont la compréhension n'en dépend pas pour autant. Sans contester l'autonomie des deux arts qu'il met en regard, il propose une appréhension globale de la vie culturelle de la Grande Grèce et de la Sicile au iv ${ }^{\mathrm{e}}$ siècle av. J.-c. Examinant chaque vase séparément, il essaie de repérer des correspondances dans la façon dont est narré le mythe dans les deux ouvres qu'il est tenté de mettre en relation. l e vase n'offre pas l'image exacte d'une représentation théâtrale mais, au mieux, le mythe tel qu'il est présenté par l'œuvre dramatique. l es images peuvent ainsi concorder avec un élément purement verbal de la pièce tel que le récit d'un messager ou l'évocation d'un rêve. l es représentations figurées de sentiments ou d'idées abstraites sont fréquentes sur la céramique apulienne, une érinye incarnant par exemple l'esprit de vengeance qui anime l'un des protagonistes. Parfois le souvenir d'une tragédie semble se superposer à une version différente du mythe, éventuellement fixée par la tradition iconographique. c omme on le voit, l'échelle de ces correspondances est très large et les conclusions de l'auteur sont d'autant plus convaincantes qu'elles sont mesurées et nuancées en fonction de chaque situation. O. taplin établit aussi une liste de signes éventuels de l'empreinte

\footnotetext{
1. Cratère à

volutes apulien attribué au peintre de Darius, avec une scène évoquant le récit du messager dans I'Hippolyte d'Euripide (détail), vers 340 av. J.-C., Londres, The British Museum.
}

d'un texte dramatique sur l'image : accessoires du costume ou du décor, présence d'un pédagogue ou d'une érinye (fig. 1), indications de noms en dialecte attique ou insertion dans l'image d'un trépied consacré à l'issue d'une représentation victorieuse.

c ette enquête vise en premier lieu à vérifier l'hypothèse qui lui sert de point de départ, à savoir que les tragédies athéniennes du v ${ }^{\mathrm{e}}$ siècle av. J.-c. étaient largement connues, car très fréquemment représentées, en Grande Grèce et en Sicile, au iv ${ }^{\mathrm{e}}$ siècle av. J.-c . Parmi les nombreux témoignages de cette diffusion ${ }^{4}$, on mentionnera seulement le cratère en cloche apulien daté des années 370 av. J.-c. incontestablement orné d'une scène des Thesmophories d'a ristophane (411 av. J.-c.) parodiant le Télèphe d'Euripide (Würzburg, Martin von Wagner Museum, H 5697). Sur les cent neuf céramiques étudiées par O. taplin, un tiers peut être mis en relation avec une tragédie non-identifiable, un tiers avec une pièce entièrement conservée d'Eschyle, Sophocle ou Euripide, un tiers avec une de leurs œuvres perdues.

un autre des enjeux majeurs de cet ouvrage est de reposer la question de la réception et de l'appréciation du théâtre grec, voire attique, par les élites italiques, dans les tombes desquelles un certain nombre de ces céramiques ont été découvertes. Beaucoup proviennent du nord de l'a pulie, de cités comme Ruvo et c anosa. On présume le plus souvent que ces vases grecs ont été acquis uniquement comme objets de prestige par des peuples considérés comme rustres et provinciaux. O. taplin souligne au contraire la grande connaissance des textes dramatiques grecs qu'impliquent ces images très élaborées dans lesquelles sont insérées des inscriptions grecques. Mais alors que l uca Giuliani fait l'hypothèse d'une élite lettrée regroupant les peintres grecs et des interprètes chargés d'expliquer à la foule ignorante, réunie pour les funérailles, le sens de ces peintures à caractère mythologique ${ }^{5}$, il postule que le théâtre demeure principalement durant tout le iv ${ }^{\mathrm{e}}$ siècle av. J.-c. un art de la représentation et que les élites italiques qui achetaient ces vases avaient une connaissance personnelle des mythes grâce aux tragédies auxquelles ils assistaient. l e corpus paestan, constitué de vases découverts dans les tombes d'élites lucaniennes fortement hellénisées, s'inscrit également dans 
cette problématique. l'enquête fait ainsi apparaître l'attrait des Paestans pour l'œuvre d'Euripide ainsi que pour les scènes tirées de l'Orestie. l'attribution fréquente de noms inconnus de la tradition littéraire à des personnages de second plan, qui contraste avec la concordance des inscriptions apuliennes avec les textes athéniens, témoigne de l'émancipation d'un peintre comme a stéas et de ses clients par rapport à la tradition littéraire athénienne.

En conclusion de son essai introductif, l'auteur revient sur la fonction que l'on suppose consolatrice de ces peintures exposées au cours des funérailles, après avoir émis l'hypothèse que le choix du sujet s'explique peut-être parfois par le goût particulier du défunt pour le théâtre tragique, voire pour une œuvre particulière. c ertains de ces vases auraient pu être des ouvres de commande. En offrant le spectacle du destin malheureux de héros mythiques, ces images apaisent la douleur des vivants à qui elle facilite l'acceptation de la simple condition humaine et de ses souffrances inhérentes. O. taplin avance cette belle idée que les œuvres tragiques picturales et littéraires offrent aussi aux humains une consolation d'ordre esthétique en donnant sens à l'expérience humaine.

l a question de la réception de la comédie grecque, voire attique, en contexte italique, constitue quant à elle l'une des problématiques majeures de publications récentes sur les vases d'italie du Sud et de Sicile présentant des scènes théâtrales à sujet comique. l es quelque deux cent cinquante vases produits en italie du Sud et en Sicile durant les trois premiers quarts du ${ }^{\text {iv }}{ }^{\mathrm{e}}$ siècle av. J.-c . sont l'objet d'un regain d'intérêt depuis la redécouverte dans les années 1980 du cratère apulien évoqué précédemment, incontestablement orné d'une scène des Thesmophories. c es vases ont été appelés à tort "phlyaques " depuis que Heydemann, en 1886, les a datés de façon erronée du iii ${ }^{e}$ siècle av. J.-c . et mis en relation avec les hilarotragédies ou "phlyaques", genre théâtral de Grande Grèce très mal connu ${ }^{6}$. il reste difficile de savoir s'ils renvoient en majorité à la représentation de comédies athéniennes jouées localement, comme le pense O. taplin, ou à la représentation de comédies locales fortement influencées par la comédie athénienne, dont il ne reste aucune trace écrite. John Richard Green utilise avec grand profit le témoignage de ces scènes

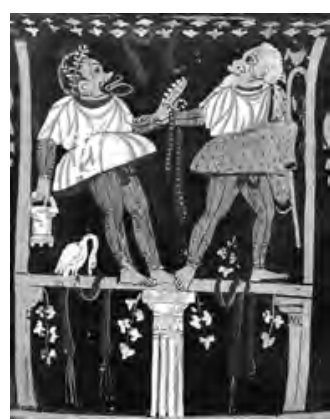

italiotes afin d'élaborer une histoire de la mise en scène comique grecque en prenant en compte ses variations régionales ${ }^{7}$. a lan Hughes cherche à déterminer, dans « c omedy in Paestan Vase Painting ", ce que les images paestanes peuvent nous apprendre à ce sujet. c e corpus iconographique, constitué d'une cinquantaine de vases, a pour particularité de ne compter que huit scènes à caractère théâtral (fig. 2) ; sur les autres, un acteur comique est représenté seul en présence de Dionysos ou participant au cortège dionysiaque (fig. 3). En se fondant sur les chronologies établies par trendall à partir de critères stylistiques, qui placent les débuts de la production paestane à sujet comique peu avant le milieu du iv ${ }^{e}$ siècle av. J.-c ., l'auteur constate un retard dans l'évolution de la mise en scène comique paestane par rapport à ce que montre la céramique sicilienne du groupe de Manfria (340-330 av. J.-c.), qu'il pense contemporaine. l a nouvelle chronologie établie par a ngela Pontrandolfo et a gnès Rouveret à la suite d'une analyse globale des mobiliers et peintures funéraires paestans se révèle néanmoins plus haute que celle de trendall et place en particulier la production comique dans la première moitié $\mathrm{du}$ iv ${ }^{\mathrm{e}}$ siècle av. J.-c . ; cela atténue considérablement le soi-disant archaïsme de la scène paestane ${ }^{8}$. Préjugeant de l'isolement $\mathrm{d}^{\prime}$ une population de langue osque, qu'il imagine peu encline aux manifestations culturelles grecques, alors que les élites lucaniennes de la région

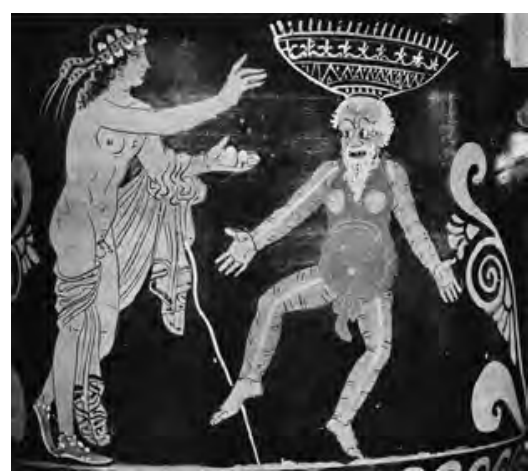

2. Cratère en cloche paestan attribué à Python, avec une scène théâtrale comique (détail), Londres, The British Museum.
3. Cratère en cloche paestan attribué à Python, avec un acteur comique dansant devant Dionysos (détail), Londres, The British Museum. 
4. Cratère en calice sicilien attribué au peintre du Louvre K 240, avec une scène dionysiaque (détail), Lipari, Museo Eoliano. étaient par exemple initiées aux théories pythagoriciennes, l'auteur forge l'hypothèse que le premier peintre paestan à représenter des scènes comiques, a steas, dont la production présente des liens iconographiques étroits avec la production sicilienne, peindrait d'après le souvenir de représentations comiques auxquelles il aurait assisté en Sicile. Ses successeurs auraient reproduit ces images théâtrales, sans avoir jamais vu une pièce de théâtre, pour des acheteurs eux-mêmes ignorants du théâtre grec. c ette hypothèse, qui repose sur un examen minutieux et bien documenté des corpus paestans et siciliens conférant à l'article son intérêt, paraît néanmoins improbable car elle néglige la question de la fonction de cette iconographie dans le contexte paestan.

l a nécessité d'une contextualisation de la céramique à sujet comique et de l'évaluation de sa signification dans un milieu indigène particulier a en effet été remarquablement illustrée par deux articles récents, l'un d'a ngela Pontrandolfo, qui cherche à définir les spécificités du corpus comique paestan, l'autre d'E. G. D. Robinson, qui pose la question de la réception du théâtre comique grec en milieu indigène apulien. Regrettant la mise en rapport mécanique des images dites "phlyaques " paestanes avec le théâtre qu'elles reflètent éventuellement, a ngela Pontrandolfo propose dans "Dioniso e personaggi fliacici..." de les analyser comme un système autonome permettant d'appréhender les représentations mentales du contexte culturel où elles sont produites. l'acteur est fréquemment représenté comme un compagnon de Dionysos, dansant ou procédant avec lui à un échange de dons, et semble faire partie intégrante du monde dionysiaque. c es images, comme celles dans lesquelles sont insérés un ou plusieurs masques comiques, témoignent d'une volonté de rendre explicite, en contexte funéraire, sur une vaisselle liée à la consommation du vin, la relation étroite existant entre le rituel dionysiaque et le théâtre. Sur ces représentations, le masque comique conserve une valeur sémantique, comme sur la céramique apulienne contemporaine, associant les mystères dionysiaques et le théâtre (fig. 4$)^{9}$. Dans un langage autonome lié à la spécificité du contexte paestan, ces images expriment, comme celle du vase attique de Pronomos, daté de la toute fin du v ${ }^{\mathrm{e}}$ siècle av. J.-c. et découvert à Ruvo, « le lien étroit entre la métamorphose qu'opère l'adoption du costume

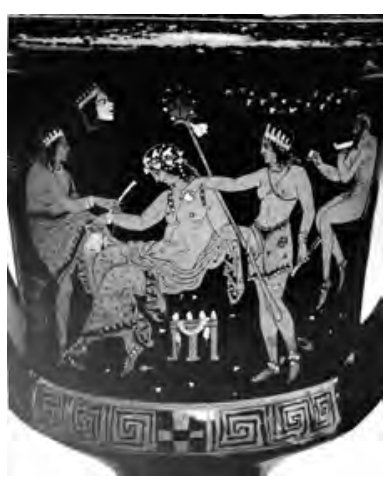

théâtral et celle qui, par le rituel de l'initiation, permet aux initiés de se transfigurer et d'entrer en communion avec la divinité " (p. 130 ${ }^{10}$. Seule la céramique paestane cependant met en scène des acteurs comiques dans le moment du rituel dionysiaque. En cherchant à articuler les programmes décoratifs des parois internes des tombes et les images figurant sur les vases qui y sont déposés, a . Pontrandolfo souligne aussi que les représentations de scènes dionysiaques dans lesquelles figure un acteur comique disparaissent de la céramique au moment où se multiplient et se diversifient dans les peintures pariétales les expressions figurées tendant à expliciter des formes de croyances eschatologiques. À côté de la représentation du passage du mort dans l'au-delà, figurent différents moments du rituel funéraire, qui semble comprendre des performances de type théâtral.

l'article d'E. G. D. Robinson, "Reception of c omic theatre...", démontre, quant à lui, que contrairement à ce qui a pu être écrit ${ }^{11}$, les populations italiques, notamment du nord de l'a pulie, s'intéressaient au théâtre comique grec et le comprenaient. il en donne pour preuve la présence de scènes en rapport avec ce théâtre sur des vases apuliens, sans doute fabriqués à tarente, de formes uniquement destinées au marché indigène. tel est le cas de trois askoi (outres) de terre cuite à figures rouges, datés du deuxième quart du iv ${ }^{e}$ siècle av. J.-c . (fig. 5), ainsi que d'un cratère à volutes sur lequel le mort est représenté sous un naiskos (petit temple), un masque comique à la main $^{12}$. En s'appuyant sur les résultats des postcolonial studies, E. G. D. Robinson souligne combien une réception passive de l'iconographie théâtrale grecque par les élites italiques est improbable. il faut au contraire envisager l'appropriation et la refonctionnalisation de ces objets. Non seulement les vases ornés de scènes comiques déposés dans les tombes devaient être considérés comme des objets de prestige qui associaient le mort aux valeurs culturelles de la métropole grecque, mais 
ils avaient aussi très probablement une fonction liée au rôle de Dionysos comme divinité de l'au-delà. Par ailleurs, dans l'italie de la seconde moitié du iv ${ }^{\mathrm{e}}$ siècle av. J.-c., qui voit naître des formes théâtrales hybrides mêlant influences grecques, étrusques et italiques, Robinson fait l'hypothèse que les scènes comiques de la céramique indigène du groupe de Xénon, dont les figures présentent des points communs avec celles du théâtre comique athénien, constitue une preuve de l'appropriation et de l'adaptation en milieu italique du modèle dramatique grec ; cela était déjà attesté par une oenochoé campanienne de Nola (British Museum F 233). Rappelant que colons et indigènes exercent les uns sur les autres une influence culturelle réciproque, E. G. D. Robinson pousse le raisonnement plus loin, non sans quelque hardiesse. contestant les conclusions d'O. taplin sur le caractère majoritairement attique des pièces de théâtre représentées sur la céramique dite "phlyaque ", l'auteur attire l'attention sur deux scènes comiques apuliennes l'une d'entre elles néanmoins nous semble de nature caricaturale plutôt que théâtrale - sur lesquelles l'un des personnages porte un costume orné de svastikas ; il s'agirait peut-être, selon lui, d'un indigène (t urin, coll. Mario Bruno ; Naples, Ma N, 118333). c es peintures renverraient alors à des pièces de théâtre locales dans lesquelles les autochtones italiens auraient constitué un sujet de moquerie naturel. l a présence de svastikas sur le rideau de scène figuré sur un vase apulien du deuxième quart du iv ${ }^{\mathrm{e}}$ siècle av. J.c. (Bari, coll. Malaguzzi-Valeri, $n^{\circ} 52$ ) pourrait

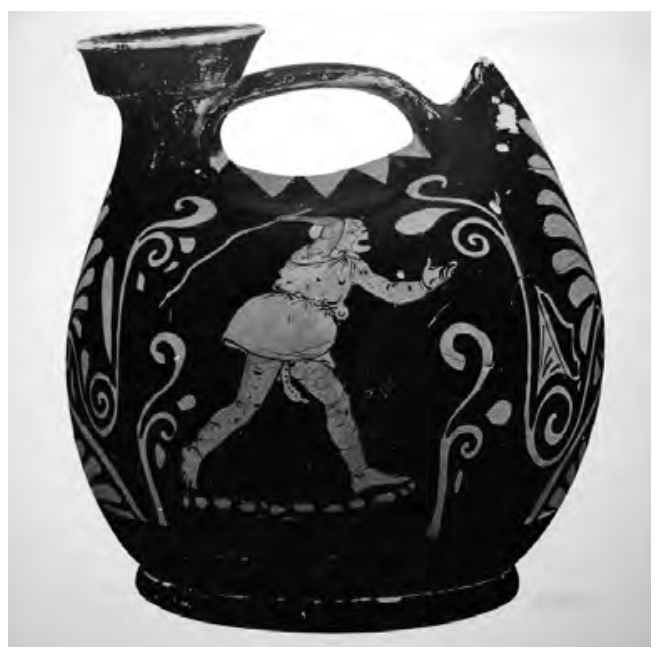

également laisser supposer, comme O. taplin en avait déjà fait l'hypothèse, que des représentations grecques avaient lieu en milieu indigène.

E. G. D. Robinson, professeur à l'u niversité de Sydney, revendique le fait que sa perception des échanges culturels entre a thènes et les cités grecques d'italie et de Sicile d'une part, entre les habitants de ces cités et les populations indigènes d'autre part, est en partie déterminée par son héritage culturel et sa propre expérience du fait colonial. c et article très stimulant met ainsi en évidence les enjeux idéologiques qui sous-tendent l'étude des questions liées à l'identité culturelle de la Grande Grèce et rappelle à quel point la reconstruction du passé est liée à la construction du présent.

\begin{abstract}
1. Près de la moitié des vases examinés ont été publiés dans les années 1970-1980 et presque tous sont répertoriés, avec deux cents autres dans 1 uigi todisco et al., La ceramica figurata a soggetto tragico in Magna Grecia e in Sicilia, Rome, 2003. cet ouvrage constitue une source d'information extrêmement utile. la question du rapport texte-image y est posée globalement, notamment dans le chapitre de c armela Roscino, " l'immagine della tragedia : elementi di caratterizzazione teatrale ed iconografia nella ceramica italiota e siceliota ", p. 223-357.
\end{abstract}

2. Oliver taplin, The Stagecraft of Aeschylus: the Dramatic Use of Exits and Entrances in Greek Tragedy, Oxford, 1977 ; idem, Greek Tragedy in Action, 1 ondres, 1978 ; idem, Comic Angels and Other Approaches to Greek Drama through Vase-Painting, Oxford, 1993.

3. I uca Giuliani, "Rhesus between Drama and Death: on the Relation of image to literature in a pulian VasePainting ", dans Bulletin of the Institute of Classical Studies, 41, 1996, p. 71-86.

4. Oliver taplin, "Spreading the Word through Performance ", dans Simon Goldhill et Robin Osborne éd., Performance Culture and Athenian Democracy, c ambridge, 1999, p. 33-57.

5. Giuliani, 1996, cité n. 3. Sur la culture des peintres apuliens, voir le bel article de Margot Schmidt, "l ivello culturale dei singoli pittori : dalla erudizione individuale all'automatismo artigianale?", dans Martine Denoyelle et al. éd., La céramique apulienne, bilan et perspectives, 2005 , p. 201-206.

6. Heinrich Heydemann, " Die Phlyakendarstellungen der bemalten Vasen ", dans Jahrbuch des Deutschen Archäologischen Instituts, 1, 1886, p. 260-313.

7. Voir notamment : " towards a Reconstruction of Performance Style ", dans Patricia Easterling, Edith Hall éd., Greek and Roman Actors, Aspects of an Ancient Profession, c ambridge, 2002, p. 93-126 ; "Smart and Stupid. the Evolution of Some Masks and characters in Fourth-
5. Askos apulien de Ruvo, vieillard courant après son esclave, vers $360-350$ av. J.-C., Malibu, The J. Paul Getty Museum. 
c entury c omedy ", dans John Davidson, a rthur Pomeroy éd., Theatres of Action. Papers for Chris Dearden, Prudentia Suppl., a uckland, 2003, p. 118-132; " the Persistent Phallos : Regional Variability in the Performance of comedy ", dans John Davidson, Frances Muecke, Peter Wilson éd. Greek Drama III, Essays in Honour of Kevin Lee, Bulletin of the Institute of Classical Studies supp. 87, 2006, p. 141-162.

8. a ngela Pontrandolfo, a gnès Rouveret, Le tombe dipinte di Paestum, 1992, p. 409 sq. ; Pon Trandolfo , 2000, p. 122 et 133-134. Contra: a rthur Dale trendall, Red Figured Vases of Paestum, l ondres, 1987, p. 19.

9. Voir John Richard Green, " $t$ heatrical Motifs in Nontheatrical c ontexts on Vases of the l ater Fifth and Fourth c enturies ", dans a lan Griffiths éd., Stage Directions, Essays in Ancient Drama in Honour of E.W. Handley, 1 ondres, 1995, p. 93-123.

10. Pon Trandolfo , 2000, p. 130 : "Il programma figurativo di questo vaso [...] esplicita la stretta connessione tra metamorfosi che opera l'adozione del costume teatrale e quella che attraverso il rituale dell'iniziazione consente agli adepti di trasfigurarsi e entrare in simbiosi con la divinità".

11. Voir par exemple John Richard Green, "the Beaulieu Painter and Provincial a pulia at the End of the Fourth c entury B.c. ", dans Elke Böhr, Wolfram Martini éd., Studien zur Mythologie und Vasenmalerei, Festschrift K. Schauenburg, Mayence, 1986, p. 181-186.

12. Ruvo, coll. Jatta 1402 ; Malibu, Getty Museum, 96. a E. 114 ; Bâle, a ntikenmuseum, Z 303 ; l ecce, Museo Provinciale, 3544 .

Alexa Piqueux, Université Paris X-Nanterre, alexa.piqueux@gmail.com

\section{l'art du portrait en Grèce et à Rome, ou de l'approche ethnique et formaliste à un phénomène culturel et politique Mario Denti}

- Wolf-Rüdiger megow, Republikanische Bildnis-Typen, Francfort-sur-le-Main/New York, Peter l ang, 2005. 155 p., 76 fig. en n. et b. iSBN : 3-631-53041-2; 48,70€.

- Massimiliano papini, Antichi volti della Repubblica. La ritrattistica in Italia centrale tra iv e ii secolo a.C., (Bullettino della Commissione Archeologica Comunale di Roma), 13, Rome, 1'Erma di Bretschneider, 2004. Vol. 1, 556 p. ; vol. 2, 200 p., 522 fig. en n. et b. iSBN : 88-8265-282-3; $140 €$.

- Peter ScHul Tz, Ralf von den Hoff éd., Early Hellenistic Portraiture. Image, Style, Context, (colloque, a thènes, institut archéologique allemand, 2002), c ambridge/New York, c ambridge university Press, 2007. 298 p., 175 fig. en n. et b. iSBN : 978-0-521-86659-0; $55 £$.

- c hristopher H. Halle TT, The Roman Nude. Heroic Portrait Statuary $200 B C-A D$ 300, Oxford, Oxford university Press, 2005. 391 p., 160 fig. en n. et b. iSBN : 978-0-19-924049$4 ; 88 £$.

l a publication récente d'une série d'ouvrages consacrés à l'art du portrait dans le monde grec et romain offre l'occasion de nous interroger sur l'état et les perspectives d'un domaine situé au cour (et au croisement) des recherches en archéologie, histoire et histoire de l'art.

l'art du portrait représente l'un des thèmes de recherche longtemps privilégié au sein de la pratique de la connaissance du monde ancien par la culture occidentale. Fortement ancré dans une dimension historico-artistique de la discipline archéologique, il a constitué à la fois une base de données irremplaçable pour les études à vocation prosopographique, un terrain particulièrement fertile pour les exégèses de type attributionniste, un répertoire iconographique et stylistique très vaste pour les analyses à caractère formel, ainsi qu'un collectage de figures_extrêmement riche, susceptible de construire des listes typologiques dépendant plus ou moins directement d'un archétype. Véritables pivots du système de communication à l'époque 